\title{
Nonlinear and time-dependent analysis of continuous unbonded prestressed concrete beams
}

\author{
Tiejiong Lou \\ CEMUC \\ Department of Civil Engineering \\ University of Coimbra \\ Coimbra 3030-788, Portugal
}

\author{
Sergio M. R. Lopes \\ CEMUC \\ Department of Civil Engineering \\ University of Coimbra \\ Coimbra 3030-788, Portugal
}

\author{
Adelino V. Lopes \\ Department of Civil Engineering \\ University of Coimbra \\ Coimbra 3030-788, Portugal
}

\begin{abstract}
This paper describes a numerical model developed to predict the full-range nonlinear response, as well as time-dependent service-load behaviour, of continuous beams prestressed with internal unbonded tendons. Both geometric and material nonlinearities are considered. The strain increment in unbonded tendon, at any deformed state, is computed from the elongation of the entire tendon between end anchorages. The unbonded prestressing contributes to the concrete beam by equivalent loads, which would be updated continuously during the solution process. The analysis reproduces the experimental results of continuous beam specimens with favourable agreement. Some important aspects of behaviour of the continuous beams are examined.
\end{abstract}

Keywords: unbonded tendons; continuous beams; numerical method; behaviour 


\section{Introduction}

In an unbonded prestressed concrete beam, there is no strain compatibility between unbonded tendons and the surrounding concrete, so that the change in the strain in unbonded tendons is member dependent rather than section dependent. Over past decades, numerous simplified approaches or empirical/semi-empirical equations for computing the stress in unbonded tendons have been proposed, including some recent studies by He and Liu [1], Yang and Kang [2], Lee and Kim [3], and Harajli [4,5]. The determination of the accurate stress in unbonded tendons should be based on the deformation of the entire tendons between anchorages, and an iterative procedure needs to be applied in the analysis. Alkhairi and Naaman [6] proposed an analytical model in which the change in strain in unbonded tendons was calculated by integrating the curvature in concrete at the level of the unbonded prestressing tendons between end anchorages. This analytical method or similar methods of analysis $[7,8]$ can predict the response of simply supported beams up to failure, but may not be able to handle continuous beams which are quite common in engineering practice.

More general numerical methods of analysis, mostly the finite element method analysis, have also been reported. Some investigators $[9,10]$ developed solid-truss models in which the concrete member was modeled by solid elements and the unbonded tendon was modeled by truss elements. Due to a large number of degrees of freedom, this model may have some limitations when applied to the analysis of large structures. For prestressed concrete beams, the beam element models have been popular for their merits of computational efficiency and accuracy. Allouche et al. [11] 
described a numerical model which used the finite beam element method incorporating an iterative moment-curvature approach to compute the stress in unbonded tendons. The model can take care of continuous rectangular beams subjected to one-point, uniform or third-point loading, but it neglected the nonlinear geometric effects of the structure which may play an important role in the behaviour of slender beams such the ones used for unbonded post-tensioned beams. Moon and Burns [12], Ariyawardena and Ghali [13], Barbieri et al. [14], Vu et al. [15] have also devoted their works to the development of numerical models for unbonded prestressed concrete beams. However, few of the models have been applied to the analysis of continuous beams prestressed with unbonded tendons.

This paper describes a numerical method for geometric and material nonlinear analysis of continuous prestressed concrete beams with internal unbonded tendons over the entire loading process up to failure. The analysis is also capable of simulating the time-dependent behaviour of such beams under service conditions. The numerical model is validated by comparing the model predictions with experimental results for continuous test beams. In addition, the proposed model is used to evaluate some important aspects of behaviour of the continuous beams, including the evolution of curvatures and neutral axis depths, the development of support reactions and bending moments, and the growth of time-dependent deformations.

\section{Finite element formulation}

Fig. 1 shows a plane beam element with two end nodes $i$ and $j$, by which the local 
coordinate system $(x, y)$ is defined. The node points are at the centroid of the concrete section. Each node has three degrees of freedom, namely, axial displacement $u$, transverse displacement $v$, and rotation $\theta$. Assuming that a plane section remains plane after bending and that the shear deformation is negligible, the axial strain $\varepsilon$ at any fibre of a concrete section is defined by [16]

$$
\varepsilon=\frac{\partial u}{\partial x}+\frac{1}{2}\left(\frac{\partial v}{\partial x}\right)^{2}-y \frac{\partial^{2} v}{\partial x^{2}}
$$

where the second term of the right side represents the large displacement effect.

Assume $u$ is a linear function and $v$ is a cubic polynomial. According to the updated Lagrangian description, the element equilibrium equations for short-term loading are as follows (inclusion of long-term or time-dependent effects is to be discussed in Section 4):

$$
\begin{aligned}
& \mathrm{d} \boldsymbol{P}^{e}=\boldsymbol{K}_{\mathrm{T}}^{e} \mathrm{~d} \boldsymbol{u}^{e}=\left(\boldsymbol{K}_{1}^{e}+\boldsymbol{K}_{2}^{e}\right) \mathrm{d} \boldsymbol{u}^{e} \\
& \boldsymbol{P}^{e}=\left\{\begin{array}{llllll}
N_{i} & V_{i} & M_{i} & N_{j} & V_{j} & M_{j}
\end{array}\right\}^{\mathrm{T}} \\
& \boldsymbol{u}^{e}=\left\{\begin{array}{llllll}
u_{i} & v_{i} & \theta_{i} & u_{j} & v_{j} & \theta_{j}
\end{array}\right\}^{\mathrm{T}} \\
& \boldsymbol{K}_{1}^{e}=\left[\begin{array}{cccccc}
d_{11} / l & 0 & -d_{12} / l & -d_{11} / l & 0 & d_{12} / l \\
& 12 d_{22} / l^{3} & 6 d_{22} / l^{2} & 0 & -12 d_{22} / l^{3} & 6 d_{22} / l^{2} \\
& & 4 d_{22} / l & d_{12} / l & -6 d_{22} / l^{2} & 2 d_{22} / l \\
& & & d_{11} / l & 0 & -d_{12} / l \\
& & & & 12 d_{22} / l^{3} & -6 d_{22} / l^{2} \\
& & & & & 4 d_{22} / l
\end{array}\right] \\
& \boldsymbol{K}_{2}^{e}=N\left[\begin{array}{cccccc}
0 & 0 & 0 & 0 & 0 & 0 \\
& 6 / 5 l & 1 / 10 & 0 & -6 / 5 l & 1 / 10 \\
& & 2 l / 15 & 0 & -1 / 10 & -l / 30 \\
& & & 0 & 0 & 0 \\
& \text { Symmetry } & & & 6 / 5 l & -1 / 10 \\
& & & & & 2 l / 15
\end{array}\right]
\end{aligned}
$$


where $\boldsymbol{P}^{e}$ is the element equivalent nodal loads; $\boldsymbol{u}^{e}$ is the element nodal displacements; $\boldsymbol{K}_{\mathrm{T}}^{e}$ is the element tangent stiffness matrix which consists of the material stiffness matrix $\boldsymbol{K}_{1}^{e}$ and the geometric stiffness matrix $\boldsymbol{K}_{2}^{e} ; l$ is the length of the beam element before deformation; and

$$
\begin{aligned}
d_{11} & =\sum_{i} E_{c i} A_{c i}+\sum_{j} E_{s j} A_{s j} \\
d_{12} & =\sum_{i} E_{c i} y_{c i} A_{c i}+\sum_{j} E_{s j} y_{s j} A_{s j} \\
d_{22} & =\sum_{i} E_{c i} y_{c i}^{2} A_{c i}+\sum_{j} E_{s j} y_{s j}^{2} A_{s j} \\
& N=\sum_{i} \sigma_{c i} A_{c i}+\sum_{j} \sigma_{s j} A_{s j}
\end{aligned}
$$

where the summation symbol signifies that the concrete section is divided into a number of layers to employ a layered approach and the subscript $c i$ represents each concrete layer and $s j$ represents each bonded reinforcement layer; $E$ is the tangent modulus for materials; $A$ corresponds to area and $\sigma$ corresponds to stress.

A load control or displacement control incremental method is used to solve the structure equilibrium equations, which are assembled in the global coordinate system from contributions of all elements. For each increment step, the Newton-Raphson iterative algorithm is employed to eliminate the out-of-balance loads:

$$
\boldsymbol{R}=\boldsymbol{P}-\boldsymbol{Q}=\boldsymbol{P}_{\text {load }}+\boldsymbol{P}_{\text {pres }}-\boldsymbol{Q}
$$

where $\boldsymbol{Q}$ is the structure internal resisting loads; and $\boldsymbol{P}$ is the structure equivalent nodal loads which consists of two components, namely, $\boldsymbol{P}_{\text {load }}$ due to external loads and $\boldsymbol{P}_{\text {pres }}$ due to unbonded prestressing. The obtainment of $\boldsymbol{P}_{\text {pres }}$ is to be discussed in the following section. 


\section{Numerical treatment of unbonded tendons}

The contribution of unbonded tendons to the concrete beam is made by transforming the prestressing force into equivalent nodal loads acting on the finite element model. It is to be noted that the equivalent loads of unbonded prestressing used in the current analysis are different from the conventional equivalent loads of bonded prestressing. For the equivalent load method of bonded prestressing, the effective prestress $f_{p e}$ is converted into the equivalent loads and then the prestressing steel are considered as ordinary bonded reinforcement with yield strength of $\left(f_{p y}-f_{p e}\right)$ and ultimate strength of $\left(f_{p u}-f_{p e}\right)$, where $f_{p y}$ and $f_{p u}$ are the yield stress and ultimate strength of prestressing steel, respectively. For unbonded prestressing, however, the preceding method is no longer applicable due to strain incompatibility between the unbonded tendons and the surrounding concrete. In this analysis, the equivalent loads of unbonded prestressing are obtained from the transformation of the current prestressing force updated consecutively during the solution process.

The unbonded prestressing tendon can be considered as an assemblage of a series of tendon segments, each of which spans a beam element. Whatever the original shape of the tendon is, as long as the length of the beam element is sufficiently small, the corresponding tendon segment can be approximated as a straight-line segment [17]. The length of the tendon segment at any deformed state can be expressed as

$$
l_{p}=\sqrt{\left(X_{p j}-X_{p i}\right)^{2}+\left(Y_{p j}-Y_{p i}\right)^{2}}
$$

where $\left(X_{p i}, Y_{p i}\right)$ and $\left(X_{p j}, Y_{p j}\right)$ are the global coordinates of the tendon segment joints $p i$ and $p j$, respectively. As shown in Fig. 2, the joints $p i$ and $p j$ of the tendon 
segment are related to the corresponding nodes $i$ and $j$ of the beam element by

$$
\begin{gathered}
X_{p i}=X_{i}-e_{i} \sin \alpha_{0}-e_{i} \theta_{i}^{g} \cos \alpha_{0} ; Y_{p i}=Y_{i}+e_{i} \cos \alpha_{0}-e_{i} \theta_{i}^{g} \sin \alpha_{0} \\
X_{p j}=X_{j}-e_{j} \sin \alpha_{0}-e_{j} \theta_{j}^{g} \cos \alpha_{0} ; Y_{p j}=Y_{j}+e_{j} \cos \alpha_{0}-e_{j} \theta_{j}^{g} \sin \alpha_{0} \\
\cos \alpha_{0}=\left(X_{j 0}-X_{i 0}\right) / l ; \sin \alpha_{0}=\left(Y_{j 0}-Y_{i 0}\right) / l
\end{gathered}
$$

in which $\left(X_{i}, Y_{i}\right)$ and $\left(X_{j}, Y_{j}\right)$ are the global coordinates of the beam element nodes $\mathrm{i}$ and $\mathrm{j}$, respectively; $\left(X_{i 0}, Y_{i 0}\right)$ and $\left(X_{j 0}, Y_{j 0}\right)$ are the global coordinates, at the original undeformed state, of the beam element nodes $i$ and $j$, respectively; $e_{i}$ and $e_{j}$ are the eccentricities of unbonded tendons at nodes $i$ and $j$, respectively; $\theta_{i}^{g}$ and $\theta_{j}^{g}$ are the rotations at nodes $i$ and $j$, respectively; and $\alpha_{0}$ is the original angle between the global coordinate axis and the local one.

As illustrated in Fig. 2, the global coordinates of the beam element nodes at any deformed state are determined by

$$
\begin{gathered}
X_{i}=X_{i 0}+u_{i}^{g} ; \quad Y_{i}=Y_{i 0}+v_{i}^{g} \\
X_{j}=X_{j 0}+u_{j}^{g} ; \quad Y_{j}=Y_{j 0}+v_{j}^{g}
\end{gathered}
$$

in which $u_{i}^{g}$ and $u_{j}^{g}$ are the global X-displacements at nodes $i$ and $j$, respectively; $v_{i}^{g}$ and $v_{j}^{g}$ are the global Y-displacements at nodes $i$ and $j$, respectively.

Combining Eqs. (9)-(12), the tendon segment length can be expressed as a function of the global nodal displacements (X, Y displacements and rotation) of the beam element. In other words, the tendon segment length, at any deformed state, can be determined in terms of the global nodal displacements. The global nodal displacement increments, and thereby the global nodal displacements, are obtained by solving the structure equivalent equation during the iterative procedure. 
The length of the tendon segment at the original undeformed state is:

$$
l_{p 0}=\sqrt{l^{2}+\left(e_{j}-e_{i}\right)^{2}}
$$

Assume that the friction forces between the unbonded tendon and the duct are negligible. Therefore, the strain in the unbonded tendon is constant over its full length, and the strain increment in the tendon, above the original state, can be calculated from the elongation of the tendon between two end anchorages:

$$
\Delta \varepsilon_{p}=\left(\sum l_{p}-\sum l_{p 0}\right) / \sum l_{p 0}
$$

where the summation is made for all tendon segments.

The total strain in unbonded tendons is then obtained from

$$
\varepsilon_{p}=\varepsilon_{p 0}+\Delta \varepsilon_{p}
$$

where $\varepsilon_{p 0}$ is the original tendon strain.

Substituting the strain $\varepsilon_{p}$ into the constitutive relationship for unbonded prestressing tendons yields the stress $\sigma_{p}$. For prestressing steel, the stress-strain relationship may be expressed using the Menegotto and Pinto equation [18] as follows:

$$
\sigma_{p}=E_{p} \varepsilon_{p}\left[Q+\frac{1-Q}{\left\{1+\left[\varepsilon_{p} E_{p} /\left(K f_{p y}\right)\right]^{R}\right\}^{1 / R}}\right]
$$

where $E_{p}$ is the modulus of elasticity of prestressing steel; $f_{p y}$ is the yield stress; and $K$, $Q$ and $R$ are the empirical parameters.

Multiply the stress $\sigma_{p}$ by the tendon area $A_{p}$ to obtain the prestressing force $N_{p}$. It is to be noted that for a time-dependent analysis, the tendon relaxation should be considered in the calculation of the tendon stress or force (to be discussed in the next section). When the prestressing force in unbonded tendons is known, as shown in 
Fig. 3, its contribution to the element equivalent nodal forces is obtained by

$$
\boldsymbol{F}_{\text {pres }}^{e}=N_{p}\left\{\begin{array}{llllll}
-\cos \gamma & \sin \gamma & e_{i} \cos \gamma & \cos \gamma & -\sin \gamma & -e_{j} \cos \gamma
\end{array}\right\}^{\mathrm{T}}
$$

where $\gamma$ is the angle between the tendon segment and the longitudinal axis of the beam element. As shown in Fig. 3,

$$
\sin \gamma=\left(e_{i}-e_{j}\right) / l_{p}
$$

The element equivalent nodal loads $\boldsymbol{P}_{\text {pres }}^{e}$ due to prestressing are exactly the opposite of the equivalent nodal forces indicated by Eq. (17). The structure equivalent nodal loads due to unbonded prestressing are assembled from the contribution of all the tendon segments by

$$
\boldsymbol{P}_{\text {pres }}=\sum \boldsymbol{T}^{\mathrm{T}} \boldsymbol{P}_{\text {pres }}^{e}
$$

where $\boldsymbol{T}$ is the displacement transformation matrix, which would be varying continuously during the solution process in accordance with the continuous change of the direction of the local coordinate system.

According to Eq. (15), the value of the tendon strain at the original undeformed state is required for the calculation of the current strain in unbonded tendons. Also, the original stress in unbonded tendons would be applied for the analysis of prestress transfer. Due to elastic compression, the stress in tendons after the prestress transfer is generally smaller than the original value. The tendon stress after the prestress transfer is referred to as the effective prestress, the value of which is usually known. The original tendon stress (strain) can be determined according to the effective prestress by the trial-and-error method as follows: (1) estimate approximately a value of the original tendon stress, and then transform it into equivalent nodal loads; (2) analyze 
the beam and, when the analysis is completed, check the difference between the tendon stress calculated and the effective prestress; (3) adjust the original tendon stress according to this stress difference, and the process is repeated until the difference vanishes.

\section{Time-dependent analysis}

The workability of a prestressed concrete structure after a long period of time is subject to unavoidable loss due to time-dependent effects such as creep and shrinkage of concrete and relaxation of prestressing tendons. The time-dependent behaviour of prestressed concrete structures under service conditions is a major interest for researchers and engineers.

The total concrete strain at time $\mathrm{t}, \varepsilon_{c}(t)$, may be expressed as follows:

$$
\varepsilon_{c}(t)=\varepsilon_{c}^{m}(t)+\varepsilon_{c}^{c r}(t)+\varepsilon_{c}^{s h}(t)
$$

where $\varepsilon_{c}^{m}(t), \varepsilon_{c}^{c r}(t)$ and $\varepsilon_{c}^{s h}(t)$ are the mechanical strain, creep strain and shrinkage strain, respectively.

The shrinkage strain is independent of the applied stress and can be determined conveniently according to design codes, while creep is associated with the history of the applied stress. Assuming that there is a linear relationship between creep and the applied stress, which is generally true under service conditions, the creep strain due to the applied stress that gradually changes with time can be expressed by applying the principle of superposition as follows:

$$
\varepsilon_{c}^{c r}(t)=\sigma_{c}\left(t_{0}\right) C\left(t, t_{0}\right)+\int_{t_{0}}^{t} C(t, \tau) \frac{\partial \sigma_{c}(\tau)}{\partial \tau} d \tau
$$


where $\sigma_{c}\left(t_{0}\right)$ and $\sigma_{c}(\tau)$ is the initial stress applied at time $\mathrm{t}_{0}$ and the stress applied at time $\tau$, respectively. $C(t, \tau)$ is the creep compliance, defined as the creep strain at time $t$ caused by a unit stress applied at time $\tau$. The form of the creep compliance adopted in this study is as follows [19]:

$$
C(t, \tau)=\sum_{k=1}^{m} \phi_{k}(\tau)\left[1-e^{-r_{k}(t-\tau)}\right]
$$

where $\mathrm{m}, \phi_{k}(\tau)$ and $r_{k}$ are the empirical parameters, which can be determined according to experimental data. The advantage of this form is its efficiency in simulating the history of the applied stress.

The time is divided into a number of small intervals. By utilizing the above form of the creep compliance, the creep strain increment at time interval $\Delta t_{n}\left(=t_{n}-t_{n-1}\right)$, $\Delta \varepsilon_{n}^{c r}$, is given by

$$
\Delta \varepsilon_{n}^{c r}=\varepsilon_{c}^{c r}\left(t_{n}\right)-\varepsilon_{c}^{c r}\left(t_{n-1}\right)=\eta_{n}+C\left(t_{n}, t_{n-1 / 2}\right) \Delta \sigma_{n}
$$

in which $t_{n-1 / 2}$ represents the middle time between time $t_{n-1}$ and time $t_{n} ; \Delta \sigma_{n}$ is the stress increment at time interval $\Delta t_{n} ;$ and

$$
\eta_{n}=\sum_{k=1}^{m}\left(1-e^{-r_{k} \Delta t_{n}}\right) \omega_{k n}
$$

where $\omega_{k n}$ is obtained from the following recursive formula:

$$
\begin{gathered}
\omega_{k n}=\omega_{k(n-1)} e^{-r_{k} \Delta t_{n-1}}+\Delta \sigma_{n-1} \phi_{k}\left(t_{(n-1)-1 / 2}\right) e^{-r_{k} \Delta t_{n-1} / 2} \\
\omega_{k 1}=\sigma_{c}\left(t_{0}\right) \phi_{k}\left(t_{0}\right)
\end{gathered}
$$

By applying the virtual work principle, the time-dependent incremental equilibrium equations, at time interval $\Delta t_{n}$, for a beam element are determined as follows: 


$$
\begin{gathered}
\Delta \boldsymbol{P}_{n}^{e}+\left(\Delta \boldsymbol{P}_{n}^{e}\right)^{c r}+\left(\Delta \boldsymbol{P}_{n}^{e}\right)^{s h}=\left(\overline{\boldsymbol{K}}_{1}^{e}+\boldsymbol{K}_{2}^{e}\right) \Delta \boldsymbol{u}_{n}^{e} \\
\left(\Delta \boldsymbol{P}_{n}^{e}\right)^{c r}=\left\{\begin{array}{llllll}
-\sum_{i} \bar{E}_{c i} A_{c i} \eta_{n i} & 0 & \sum_{i} \bar{E}_{c i} y_{c i} A_{c i} \eta_{n i} & \sum_{i} \bar{E}_{c i} A_{c i} \eta_{n i} & 0 & \left.-\sum_{i} \bar{E}_{c i} y_{c i} A_{c i} \eta_{n i}\right\}^{\mathrm{T}} \\
\left(\Delta \boldsymbol{P}_{n}^{e}\right)^{s h}=\Delta \varepsilon_{n}^{s h}\left\{-\sum_{i} \bar{E}_{c i} A_{c i}\right. & 0 & \sum_{i} \bar{E}_{c i} y_{c i} A_{c i} & \sum_{i} \bar{E}_{c i} A_{c i} & 0 & \left.-\sum_{i} \bar{E}_{c i} y_{c i} A_{c i}\right\}^{\mathrm{T}}
\end{array}\right.
\end{gathered}
$$

where $\Delta \boldsymbol{P}_{n}^{e}$ is the equivalent nodal load increments due to external loads and unbonded prestressing; $\left(\Delta \boldsymbol{P}_{n}^{e}\right)^{c r}$ and $\left(\Delta \boldsymbol{P}_{n}^{e}\right)^{s h}$ represent the equivalent nodal load increments due to concrete creep and concrete shrinkage, respectively; $\Delta \varepsilon_{n}^{s h}$ is the shrinkage strain increment; $\Delta \boldsymbol{u}_{n}^{e}$ is the nodal displacement increments; the form of the material stiffness matrix $\overline{\boldsymbol{K}}_{1}^{e}$ is a modification of $\boldsymbol{K}_{1}^{e}$ by replacing the coefficients $d_{11}, d_{12}$ and $d_{22}$ in Eq. (5a) with the coefficients $\bar{d}_{11}, \bar{d}_{12}$ and $\bar{d}_{22}$, respectively; the form of the geometric stiffness matrix $\boldsymbol{K}_{2}^{e}$ expressed by Eq. (5b) is also used here.

$$
\begin{gathered}
\bar{d}_{11}=\sum_{i} \bar{E}_{c i} A_{c i}+\sum_{j} E_{s j} A_{s j} \\
\bar{d}_{12}=\sum_{i} \bar{E}_{c i} y_{c i} A_{c i}+\sum_{j} E_{s j} y_{s j} A_{s j} \\
\bar{d}_{22}=\sum_{i} \bar{E}_{c i} y_{c i}^{2} A_{c i}+\sum_{j} E_{s j} y_{s j}^{2} A_{s j} \\
\bar{E}_{c}=\bar{E}_{c}\left(t_{n-1 / 2}\right)=\frac{E_{c}\left(t_{n-1 / 2}\right)}{1+C\left(t_{n}, t_{n-1 / 2}\right) E_{c}\left(t_{n-1 / 2}\right)}
\end{gathered}
$$

In the calculation of the tendon stress, which would be transformed into equivalent loads as described previously, the prestress loss due to tendon relaxation should be considered. In this study, the relaxation of prestressing steel, $\Delta \sigma_{p r}$, is computed based on the following equation [20]:

$$
\frac{\Delta \sigma_{p r}}{\sigma_{p 0}}=-\frac{\log \left(\tau-t_{0}\right)}{10}\left(\frac{\sigma_{p 0}}{f_{p y}}-0.55\right)
$$

in which $\left(\tau-t_{0}\right)$ is the time in hours after stressing. This equation is subject to the 
condition that $\sigma_{p 0}$ (original stress immediately after stressing) is the only applied stress and the tendon length is kept constant. In a prestressed concrete beam, the applied stress would be affected by some causes such as prestress transfer, the application of external loads, creep and shrinkage of concrete. Therefore, the original stress used to calculate the relaxation at each time interval should be modified according to the change in the applied stress due to these causes. The total relaxation at time $\mathrm{t}_{\mathrm{n}}$ is obtained by summing up the relaxation at all time intervals.

\section{Application}

\subsection{Continuous test beams and analysis model}

In an experimental programme at the structural laboratory of Hunan University [21], a series of continuous beams post-tensioned with internal unbonded tendons were tested to failure. Five of the beams, designated as YLA1, YLA2, YLB2, YLC1 and YLC2 are used to calibrate the proposed model. The dimensions and steel layouts are shown in Fig. 4. The beams were of a rectangular section with width of $150 \mathrm{~mm}$ and depth of $300 \mathrm{~mm}$, and were continuous over two spans (4800 $\mathrm{mm}$ each span). Both spans were under third-point loading. The layout of the unbonded tendons was kept constant for all beams and was defined by the following equations:

$$
\begin{aligned}
& y^{*}=2.5297 \times 10^{-5} x^{* 2}-0.1006 x^{*} \text { for } 0 \leq x^{*} \leq 1988 \mathrm{~mm} \\
& y^{*}=3.0518 \times 10^{-5} x^{* 2}-0.1214 x^{*}+20.7 \text { for } 1988 \mathrm{~mm}<x^{*} \leq 4320 \mathrm{~mm} \\
& y^{*}=-1.4817 \times 10^{-4} x^{* 2}+1.4225 x^{*}-3313.9 \text { for } 4320 \mathrm{~mm}<x^{*} \leq 4800 \mathrm{~mm}
\end{aligned}
$$

where $x^{*}$ is the distance to the end support of the beams; and $y^{*}$ is the distance 
between the tendon line and the centroidal axis of the beams. The tendon lines outside the end supports were straight lines tangent to the tendon curve represented by Eq. (31a) at $x^{*}=0$.

The unbonded prestressing steel consisted of $15 \mathrm{~mm}$ seven-wire strand having ultimate strength, yield strength and elastic modulus of $1941 \mathrm{MPa}, 1680 \mathrm{MPa}$ and 1.97 GPa, respectively. The longitudinal nonprestressed reinforcement consisted of 12 , 16 or $18 \mathrm{~mm}$ deformed steel bars having yield strengths of 361,384 and $364 \mathrm{MPa}$, respectively. The shear reinforcement consisted of $10 \mathrm{~mm}$ stirrups with spacing of 150 $\mathrm{mm}$ in the outer shear spans and of $200 \mathrm{~mm}$ in the flexural spans for all beams, while the shear reinforcement in the inner shear spans consisted of $10 \mathrm{~mm}$ stirrups with spacing of $150 \mathrm{~mm}$ for Beams YLA1 and YLA2, $100 \mathrm{~mm}$ for Beam YLB2 and $80 \mathrm{~mm}$ for Beams YLC1 and YLC2. The amount of nonprestressed steel $\left(A_{s 1}, A_{s 2}, A_{s 3}\right.$ and $\mathrm{A}_{\mathrm{s} 4}$ ), the concrete strength $f_{c}^{\prime}$ and the effective prestress $f_{p e}$ for the beams are given in Table 1.

In the finite element idealization, the concrete beam is idealized as 38 beam elements, and the cross section of a beam element is subdivided into 10 concrete layers and 2 steel layers each of which represents the top or bottom steel bars, as shown in Fig. 5. The unbonded tendon is also divided into 38 segments corresponding to the beam elements. The material models adopted are as follows: the Scott et al. model [22] is adopted for concrete in compression while the bilinear elastic-softening model [23] is adopted for concrete in tension (Fig. 6(a)); the Menegotto and Pinto model [18] is adopted for prestressing steel (Fig. 6(b)); and the elastic-perfectly 
plastic model is adopted for nonprestressed steel (Fig. 6(c)). During the analysis, when the concrete strain at the critical section reaches the ultimate compressive strain, the beam is assumed to be crushed.

\subsection{Comparisons between computational and experimental results}

According to the model prediction, all the beams analyzed fail by crushing of concrete at the span critical section. Prior to failure, the beams experience sequentially four typical phases, namely, first cracking at the centre support, second cracking at the span critical section, first yielding (of nonprestressed steel) at the centre support, and second yielding at the span critical section. The predicted failure mode, as well as the typical phases experienced, is consistent with the experimental observation.

Fig. 7 compares the predicted load-deflection responses with the experimental results for the test beams. It can be seen that, for Beams YLB2, YLC1 and YLC2 which had coincident experimental values between left and right spans, the computational and experimental results are in good agreement. For Beams YLA1 and YLA2, the numerical prediction agrees favorably with the experimental results for the left span, which, however, were not well coincident with those for the right span.

Fig. 8 shows the comparisons between numerical and experimental results regarding the load versus stress increase in unbonded tendons for the test beams. The experimental values of the tendon force were read from the load cells placed at the left and right stressing ends, while there existed some reading disparities between the left and right cells as can be observed in Fig. 8. It is seen that, at the initial loading stage, the analysis overestimates the stress increase in unbonded tendons, attributed to 
that the friction loss, which is rather important for the small stress increment developed at this stage, is neglected in the analysis. After cracking, the tendon stress increases quickly, and therefore the influence of the friction loss gradually diminishes. In the post-elastic range, the results obtained from the analysis agree well with the experimental results, as shown in Fig. 8 .

\subsection{Evolution of curvatures and neutral axis depths}

Fig. 9 shows the curvature evolution at the span critical section and centre support section with the applied load for the three continuous beams having different amounts of nonprestressed steel (YLA2, YLB2 and YLC1). It can be observed that, before yielding at the span critical section, the curvature at the centre support develops faster than that that at the span critical section, while it is opposite after yielding. It is also seen that, with increasing amount of nonprestressed steel, the ultimate load increases obviously, while the curvature ductility gradually decreases.

Under the prestressing force and self-weight (nil live load), there is a small hogging (sagging) curvature at the span critical section (centre support). At this state, the neutral axis lies someplace at the top (bottom) half of the span critical section (centre support). With the application and increase of the live load, the hogging (sagging) curvature at the span critical section (centre support) gradually vanishes, and then sagging (hogging) curvature begins to appear and develop. Accordingly, the neutral axis at the span critical section (centre support) moves upward (downward) to a place far above (below) the top (bottom) fibre, and suddenly jumps to a place far below (above) the bottom (top) fibre and then move upward (downward). Fig. 10 
shows the evolution of neutral axis depths at the span critical section and centre support section, after the neutral axis enters the outermost tensile fibre, with the applied load. It is seen that, before yielding at the span critical section, the neutral axis at the span critical section moves slower than that at the centre support. After yielding, the neutral axis at the span critical section moves rapidly, much faster than that at the centre support. It is also seen that the neutral axis depth increases as the amount of nonprestressed steel increases.

\subsection{Development of support reactions and bending moments}

Fig. 11 shows the development of the reactions at end and centre supports with the applied load. Both the actual and elastic values are illustrated. The actual values are obtained from the nonlinear computer analysis proposed in this study, while the elastic values are obtained from an elastic analysis. It can be seen from the figure that the actual values are identical to the elastic ones at the initial loading up to cracking. Thereafter, the actual values begin to deviate from the elastic ones due to redistribution of internal forces. Because the first cracking appears at the centre support, on cracking the internal forces are redistributed towards the critical region in the span. As a consequence, the actual reactions grow faster at the end support while slower at the centre support, as shown in Fig. 11. The reaction evolution stabilizes until the yielding of nonprestressed steel over the centre support, which leads to a further growth (diminution) of the rate of increase in the actual reactions at the end support (centre support). When the nonprestressed steel at the span critical section begins to yield, the internal forces turns to be redistributed towards the centre support, 
thereby resulting in a diminution (growth) of the rate of increase in the actual reactions at the end support (centre support). It is also observed that the difference between the actual reaction and elastic value is less obvious for higher amount of nonprestressed steel. The above observations are consistent with another experimental study [24].

Fig. 12 shows the development of the bending moments, obtained from the nonlinear and elastic analyses, at the span critical section and centre support with the applied load. It is seen that, similar to the actual reaction evolution, the actual moment evolution during the whole loading process is mainly controlled by three phases, namely, first cracking, first yielding and second yielding. Due to redistribution of moments, the load-moment relationship losses its linearity on first cracking, which causes a diminution (growth) of the rate of increase in moments at the centre support (span critical section). This change in the moment evolution is accentuated by first yielding, and later alleviated by second yielding. The degree of moment redistribution can be expressed as: $\beta=1-M / M_{e}$, where $M$ is the actual moment in the inelastic range, and $M_{e}$ is the elastic moment. It can be observed that the degree of moment redistribution decreases as the amount of nonprestressed steel increases. At the ultimate limit state, the values of $\beta$ for YLA2, YLB2 and YLC1 are respectively $27.5 \%, 22.5 \%$ and $19.1 \%$ at the centre support, and $-12.7 \%,-10.8 \%$ and $-9.5 \%$ at the span critical section.

\subsection{Growth of time-dependent deformations}

A time-dependent analysis is conducted taking into account the creep and 
shrinkage of concrete and the relaxation of prestressing steel. The values of the creep parameters defined in Eq. (22) and the time-dependent modulus of elasticity of concrete, recommended by Zhu [19] for preliminary design of large concrete dams, are slightly modified for the current analysis: $\mathrm{m}=2 ; \phi_{1}(\tau)=0.69\left(1+9.2 \tau^{-0.45}\right) / E(\tau)$, $\mathrm{r}_{1}=0.05 ; \phi_{2}(\tau)=1.56\left(1+1.7 \tau^{-0.45}\right) / E(\tau), \mathrm{r}_{2}=0.005 ; \quad E(\tau)=E_{0}\left(1-e^{-0.4 \tau^{0.34}}\right)$, where $\mathrm{E}_{0}$ is a constant, the value of which is 1.4 times the instantaneous modulus of elasticity at age of 28 days. The concrete shrinkage model recommended by MC10 [25] is adopted. It is assumed that the tendons were tensioned to an original prestressing force and then anchored to the concrete beam at concrete age of 28 days.

At zero live load (self-weight load is considered), the growth of the upward deflection (camber) at midspan and axial shortening from day 28 to day 600 due to time-dependent effects for the three beams is illustrated in Fig. 13. It can be observed that after prestress transfer, the camber increases very quickly at early age and tends to stabilize about 40 days later. The increase in axial shortening is also rapid at early age, and becomes rather slow after several hundred days. It is also observed that the nonprestressed steel has important influence on the development of time-dependent deformations, particularly of the beam camber. A higher amount of nonprestressed steel registers lower increases in the camber and axial shortening. At a service load of $60 \mathrm{kN}$, the growth of the downward deflection at midspan and axial shortening for the three beams is shown in Fig. 14. The instantaneous deflections of the beams due to the live load are $3.4 \sim 3.7 \mathrm{~mm}$, while the instantaneous axial shortenings are not noticeable, as can be seen in Fig. 14. Similar to the case of nil live load, the 
time-dependent deformations of the beams under live loading develop rapidly at first, while the development slows down with time. At given age, the higher the amount of nonprestressed steel, the lower the deflection and axial shortening.

\section{Conclusions}

A numerical model based on the finite element method is developed to predict the complete nonlinear response of continuous concrete beams prestressed with internal unbonded tendons. The model is also capable of predicting the long-term service-load behaviour of such beams due to concrete creep, concrete shrinkage and tendon relaxation. The finite element formulation is established using the layered Euler-Bernoulli beam theory. According to the updated Lagrangian description, the stiffness matrix consists of the material stiffness matrix, which represents the material nonlinear effect, and the geometric stiffness matrix, which represents the large displacement effect. The tendon strain increment, above the reference strain, is computed from the elongation of the entire tendon between end anchorages. This increment is added to the reference strain to obtain the current total strain, and thereby the tendon stress and prestressing force. The contribution of unbonded tendons to the concrete beam is made by transforming the current prestressing force into equivalent nodal loads acting on finite element model. The time-dependent effects, including concrete creep, concrete shrinkage and tendon relaxation, are introduced in the model, where concrete creep is modeled assuming a linear creep law and utilizing an efficient form of creep compliance. 
The nonlinear model is verified with the experimental results of some continuous unbonded prestressed concrete beam specimens available in literature. Comparisons between the computational and experimental results show that the numerical analysis reproduces well the experimental load-deflection response and stress increase in unbonded tendons over the entire loading range up to failure. Some important aspects of behaviour of the continuous beams are evaluated using the proposed model. The analysis shows that the amount of nonprestressed steel significantly affects the behaviour of continuous beams, including the curvature, neutral axis depth, moment redistribution and the time-dependent deformations.

\section{Acknowledgements}

The work presented in this paper has been supported by the Portuguese Foundation for Science and Technology under Grant No. SFRH/BPD/66453/2009.

\section{References}

[1] He ZQ, Liu Z. Stresses in external and internal unbonded tendons: Unified methodology and design equations. ASCE Journal of Structural Engineering 2010; 136(9): 1055-1065.

[2] Yang KH, Kang THK. Equivalent strain distribution factor for unbonded tendon stress at ultimate. ACI Structural Journal 2011; 108(2): 217-226.

[3] Lee DH, Kim KS. Flexural strength of prestressed concrete members with unbonded tendons. Structural Engineering and Mechanics 2011; 38(5): 675-696. 
[4] Harajli MH. Proposed modification of AASHTO-LRFD for computing stress in unbonded tendons at ultimate. ASCE Journal of Bridge Engineering 2011; 16(6): 828-838.

[5] Harajli MH. Tendon stress at ultimate in continuous unbonded post-tensioned members: Proposed modification of ACI 318, Eq. (18-4) and (18-5). ACI Structural Journal 2012; 109(2): 183-192.

[6] Alkhairi FM, Naaman AE. Analysis of beams prestressed with unbonded internal or external tendons. ASCE Journal of Structural Engineering 1993; 119(9): 2680-2699.

[7] Du JS, Au FTK, Cheung YK, Kwan AKH. Ductility analysis of prestressed concrete beams with unbonded tendons. Engineering Structures 2008; 30: 13-21.

[8] Ozkul O, Nassif H, Tanchan P, Harajli M. Rational approach for predicting stress in beams with unbonded tendons. ACI Structural Journal 2008; 105(3): 338-347.

[9] Wu XH, Otani S, Shiohara H. Tendon model for nonlinear analysis of prestressed concrete structures. ASCE Journal of Structural Engineering 2001; 127(4): $398-405$.

[10] Vecchio FJ, Gauvreau P, Liu K. Modeling of unbonded post-tensioned concrete beams critical in shear. ACI Structural Journal 2006; 103(1): 57-64.

[11] Allouche EN, Campbell TI, Green MF, Soudki KA. Tendon stress in continuous unbonded prestressed concrete members-Part2: Parametric study. PCI Journal 1999; 44(1): 60-73.

[12] Moon JH, Burns NH. Flexural behavior of member with unbonded tendons. I: 
Theory. ASCE Journal of Structural Engineering 1997; 123(8): 1087-1094.

[13] Ariyawardena N, Ghali A. Prestressing with unbonded internal or external tendons: analysis and computer model. ASCE Journal of Structural Engineering 2002; 128(12): 1493-1501.

[14]Barbieri RA, Gastal FPSL, Filho AC. Numerical model for the analysis of unbonded prestressed members. ASCE Journal of Structural Engineering 2006; 132(1): 34-42.

[15] Vu NA, Castel A, Francois R. Response of post-tensioned concrete beams with unbonded tendons including serviceability and ultimate state. Engineering Structures 2010; 32: 556-569.

[16]Lou TJ, Xiang YQ. Finite element modeling of concrete beams prestressed with external tendons. Engineering Structures 2006; 28(14): 1919-1926.

[17]Kang YJ, Scordelis AC. Nonlinear analysis of prestressed concrete frames. ASCE Journal of the Structural Division 1980; 106(ST2): 445-462.

[18]Menegotto M, Pinto PE. Method of analysis for cyclically loaded reinforced concrete plane frames. IABSE preliminary report for symposium on resistance and ultimate deformability of structures acted on well-defined repeated loads, Lisbon; 1973. p 15-22.

[19]Zhu BF. The finite element method theory and application. China Water Power Press, Beijing; 3rd Edition, 2009. (in Chinese)

[20]Magura DD, Sozen MA and Siess CP. A study of stress relaxation in prestressing reinforcement. PCI Journal 1964; 9(2): 13-57. 
[21]Chen YW. The experimental researches on unbonded partially prestressed concrete continuous beams. Master's thesis, Hunan University, Changsha; 2008. (in Chinese)

[22] Scott BD, Park R, Priestley MJN. Stress-strain behavior of concrete confined by overlapping hoops at low and high strain rates. ACI Journal 1982; 79(1): 13-27.

[23] Kwak HG, Kim SP. Nonlinear analysis of RC beams based on moment-curvature relation. Computers and Structures 2002; 80: 615-628.

[24]Zhou W, Zheng WZ. Experimental research on plastic design method and moment redistribution in continuous concrete beams prestressed with unbonded tendons. Magazine of Concrete Research 2010; 62(1): 51-64.

[25]Fib. Model Code 2010 (MC10). International Federation for Structural Concrete (fib). Lausanne, Switzerland; Bulletins 55 and 56, 2012. 
Table 1 Design parameters of test beams [21]

\begin{tabular}{|c|c|c|c|c|c|c|}
\hline Beam & $\begin{array}{c}\mathrm{A}_{\mathrm{s} 1} \\
\left(\mathrm{~mm}^{2}\right)\end{array}$ & $\begin{array}{c}\mathrm{A}_{\mathrm{s} 2} \\
\left(\mathrm{~mm}^{2}\right)\end{array}$ & $\begin{array}{c}\mathrm{A}_{\mathrm{s} 3} \\
\left(\mathrm{~mm}^{2}\right)\end{array}$ & $\begin{array}{c}\mathrm{A}_{\mathrm{s} 4} \\
\left(\mathrm{~mm}^{2}\right)\end{array}$ & $\begin{array}{c}f_{c}^{\prime} \\
(\mathrm{MPa})\end{array}$ & $\begin{array}{c}f_{p e} \\
(\mathrm{MPa})\end{array}$ \\
\hline YLA1 & $\begin{array}{r}452.4 \\
(4 \varnothing 12)\end{array}$ & $\begin{array}{c}226.2 \\
(2 \varnothing 12)\end{array}$ & $\begin{array}{c}226.2 \\
(2 \varnothing 12)\end{array}$ & $\begin{array}{c}226.2 \\
(2 \varnothing 12)\end{array}$ & 34.9 & 1083 \\
\hline YLA2 & $\begin{array}{r}452.4 \\
(4 \varnothing 12)\end{array}$ & $\begin{array}{c}226.2 \\
(2 \varnothing 12)\end{array}$ & $\begin{array}{c}226.2 \\
(2 \varnothing 12)\end{array}$ & $\begin{array}{c}226.2 \\
(2 \varnothing 12)\end{array}$ & 36.7 & 1196 \\
\hline YLB2 & $\begin{array}{c}603.2 \\
(3 \varnothing 16)\end{array}$ & $\begin{array}{c}508.9 \\
(2 \varnothing 18)\end{array}$ & $\begin{array}{c}226.2 \\
(2 \varnothing 12)\end{array}$ & $\begin{array}{r}603.2 \\
(3 \varnothing 16)\end{array}$ & 33.0 & 1193 \\
\hline YLC1 & $\begin{array}{r}763.4 \\
(3 \varnothing 18)\end{array}$ & $\begin{array}{c}763.4 \\
(3 \varnothing 18)\end{array}$ & $\begin{array}{c}226.2 \\
(2 \varnothing 12)\end{array}$ & $\begin{array}{r}763.4 \\
(3 \varnothing 18)\end{array}$ & 37.1 & 1169 \\
\hline YLC2 & $\begin{array}{l}763.4 \\
(3 \varnothing 18)\end{array}$ & $\begin{array}{c}763.4 \\
(3 \varnothing 18)\end{array}$ & $\begin{array}{c}226.2 \\
(2 \varnothing 12)\end{array}$ & $\begin{array}{r}763.4 \\
(3 \varnothing 18)\end{array}$ & 33.2 & 1205 \\
\hline
\end{tabular}




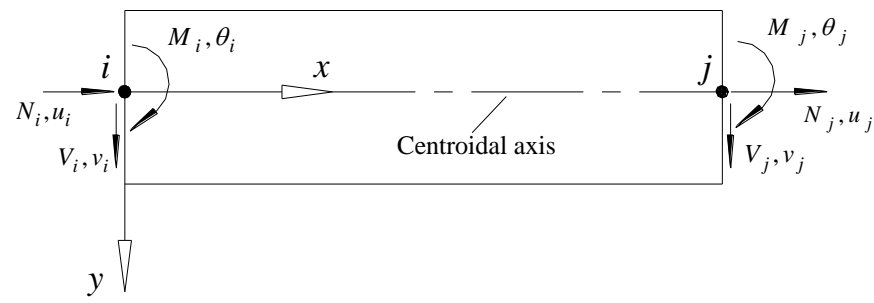

Fig. 1 Sketch of beam element 


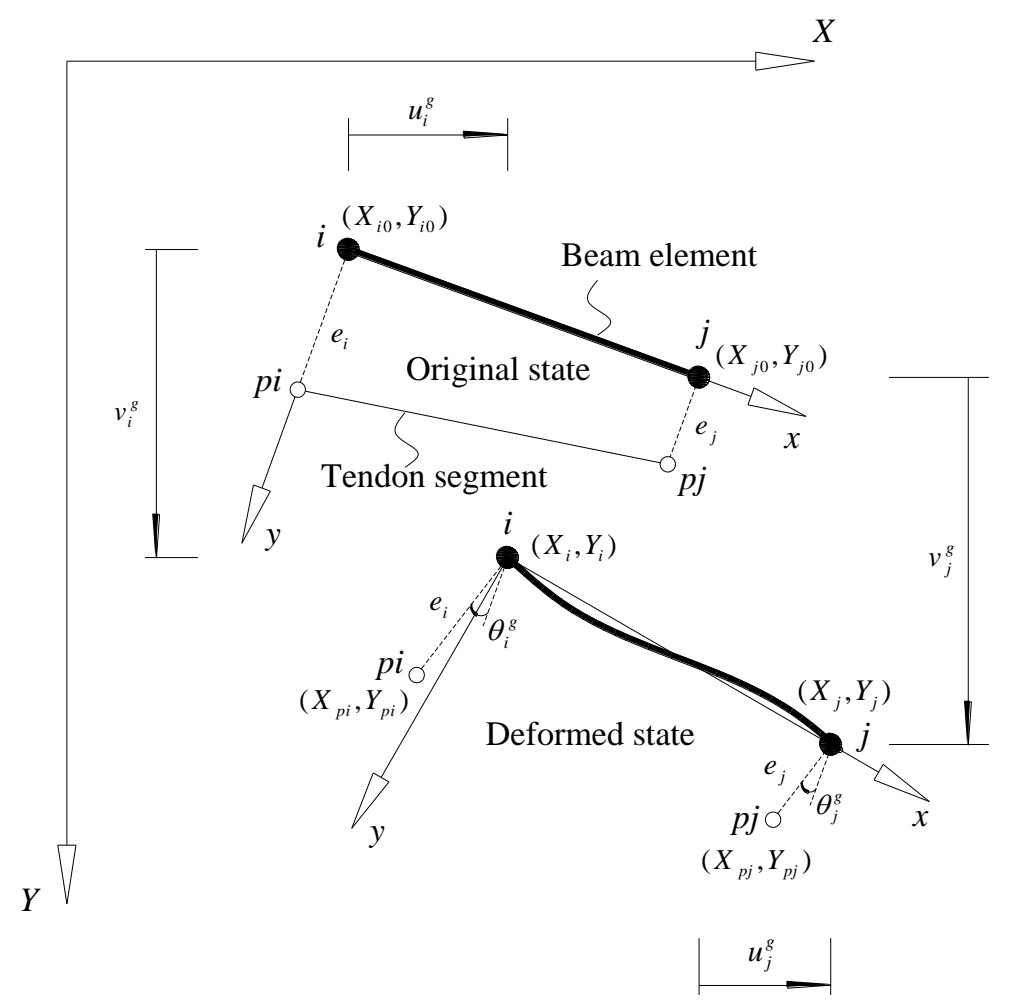

Fig. 2 Relationship between beam element nodes and tendon segment joints 


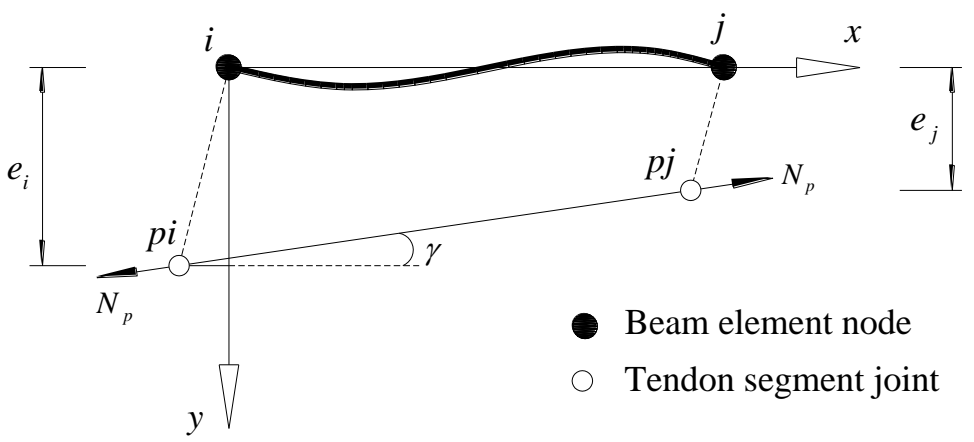

Fig. 3 Contribution of unbonded prestressing force to element nodal loads 


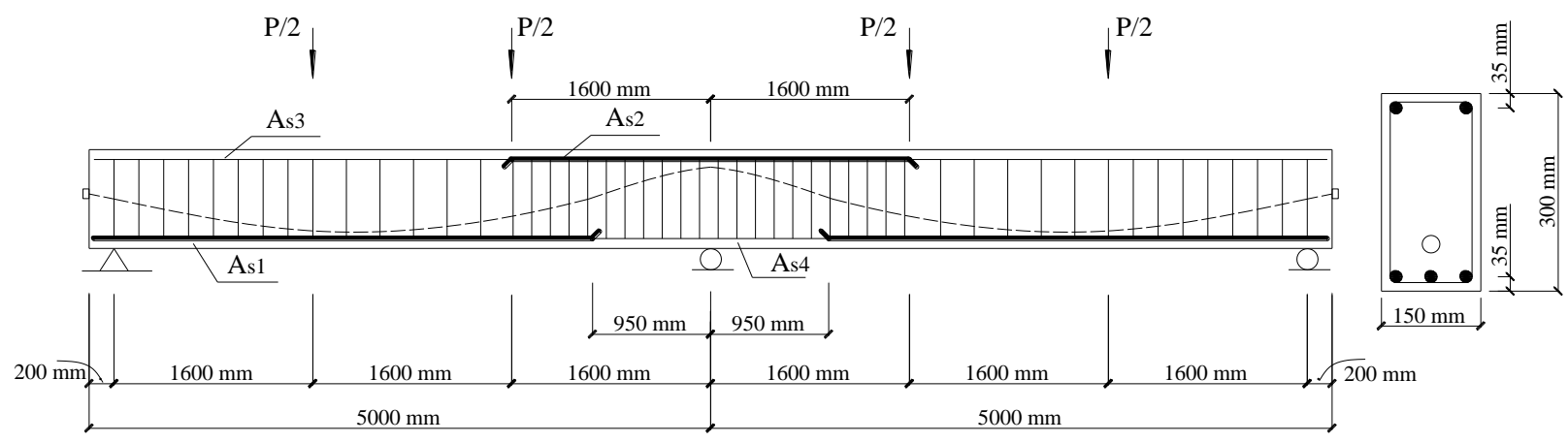

Fig. 4 Details of test beams [21] 


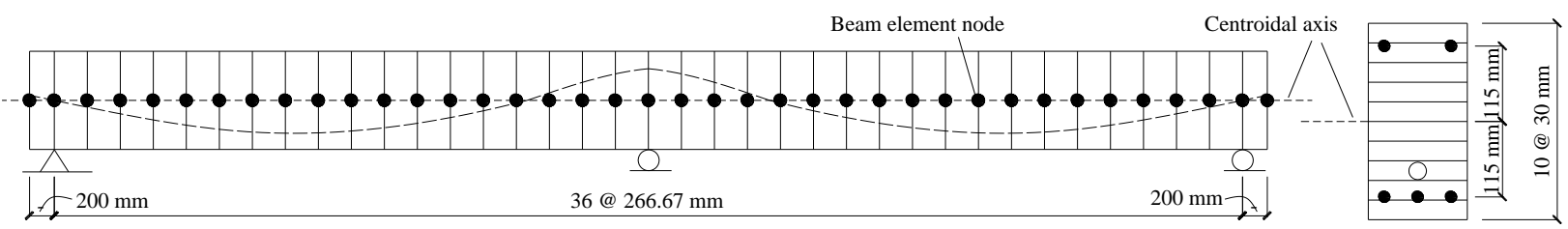

Fig. 5 Finite element model of the test beams 

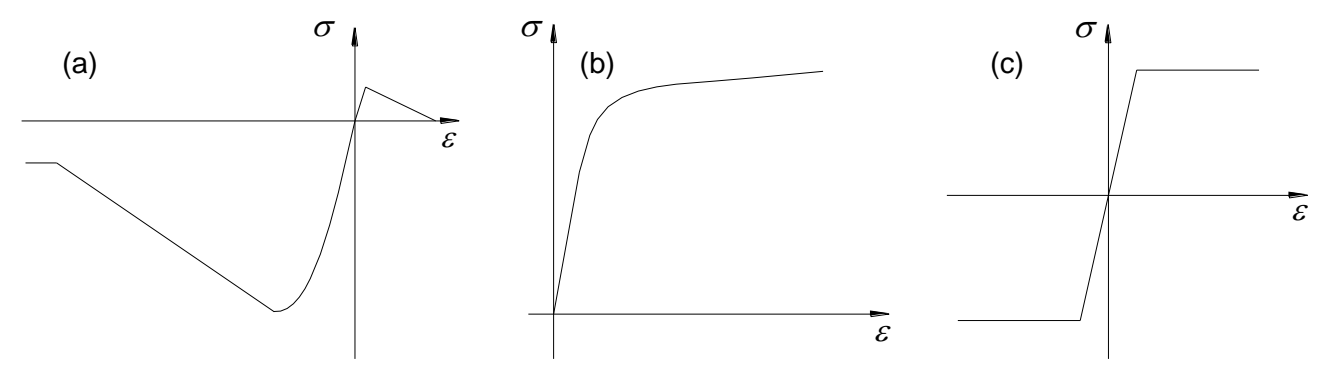

Fig. 6 Stress-strain diagrams for materials: (a) concrete; (b) prestressing steel; (c) nonprestressed steel 

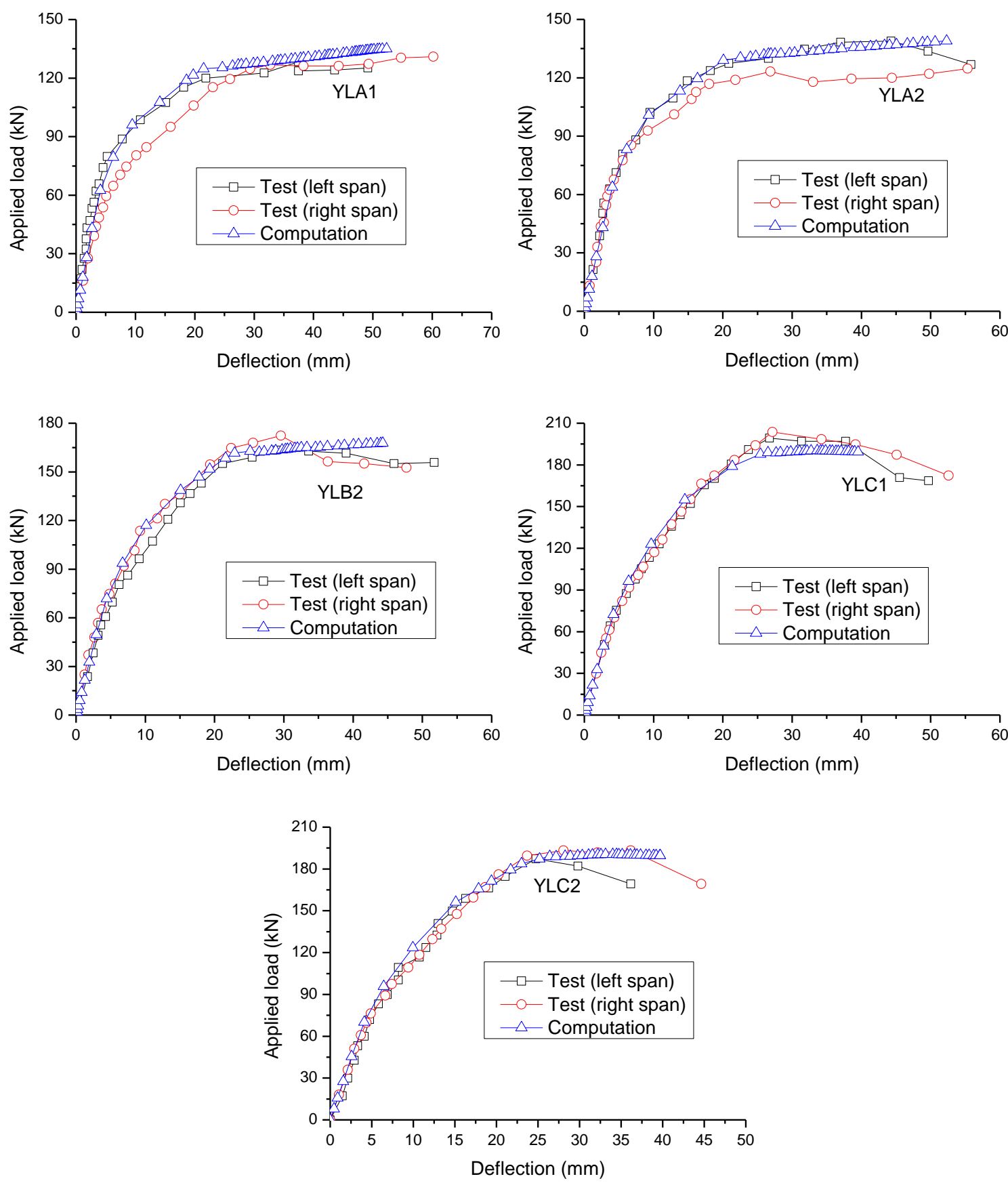

Fig. 7 Comparisons between predicted load-deflection response and experimental results for the test beams 

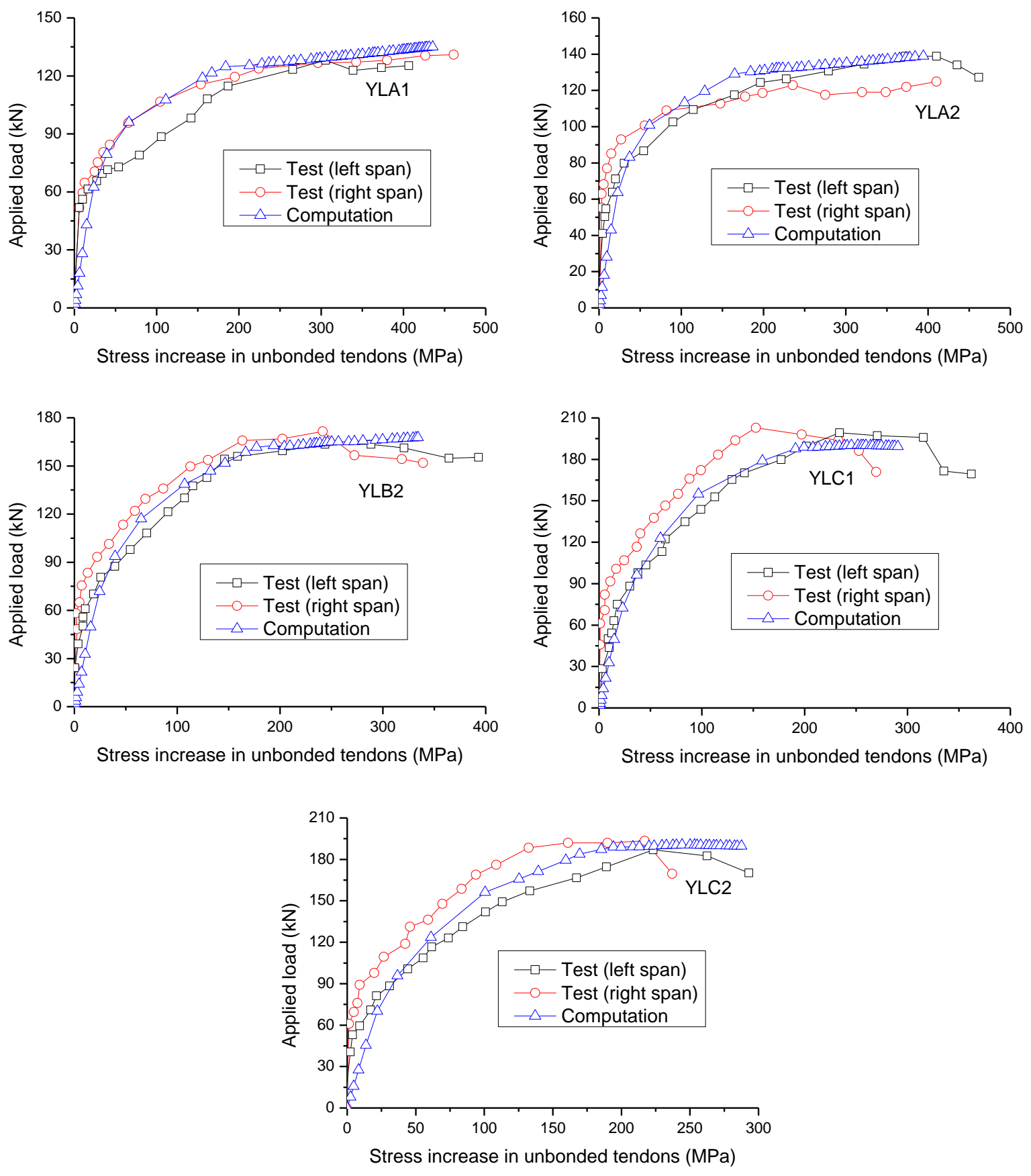

Fig. 8 Comparisons between predicted tendon stress increase and experimental results for the test beams 


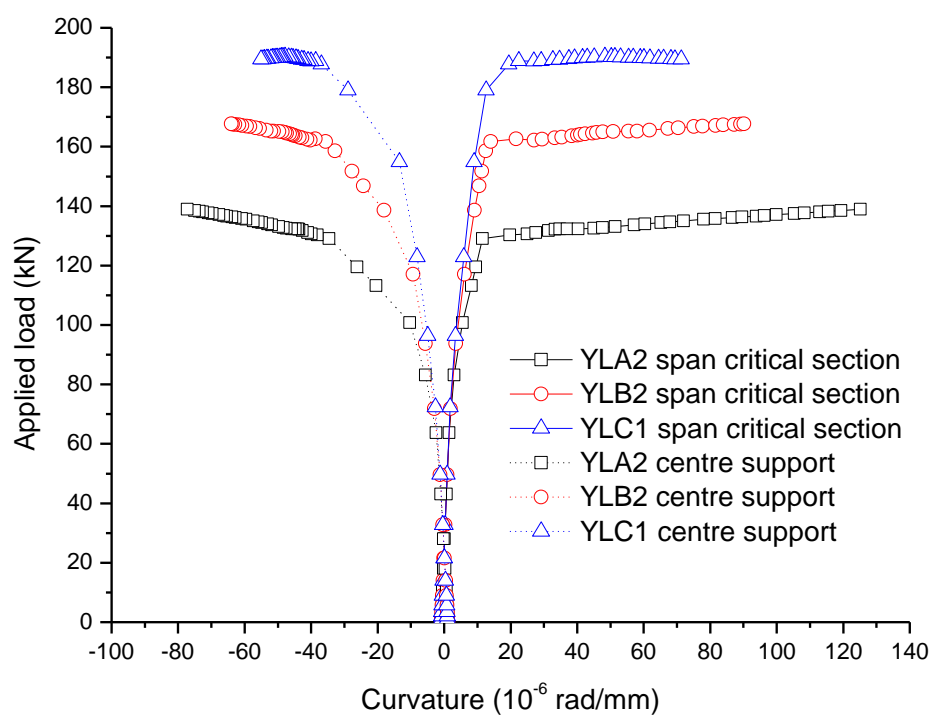

Fig. 9 Curvature evolution for the continuous beams 


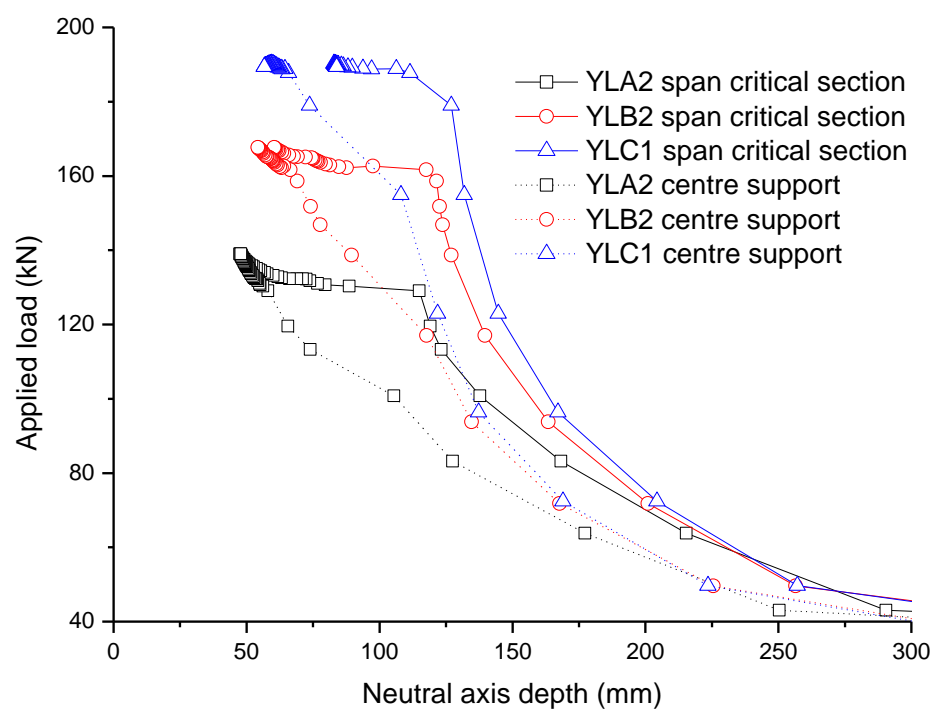

Fig. 10 Neutral axis depth evolution for the continuous beams 

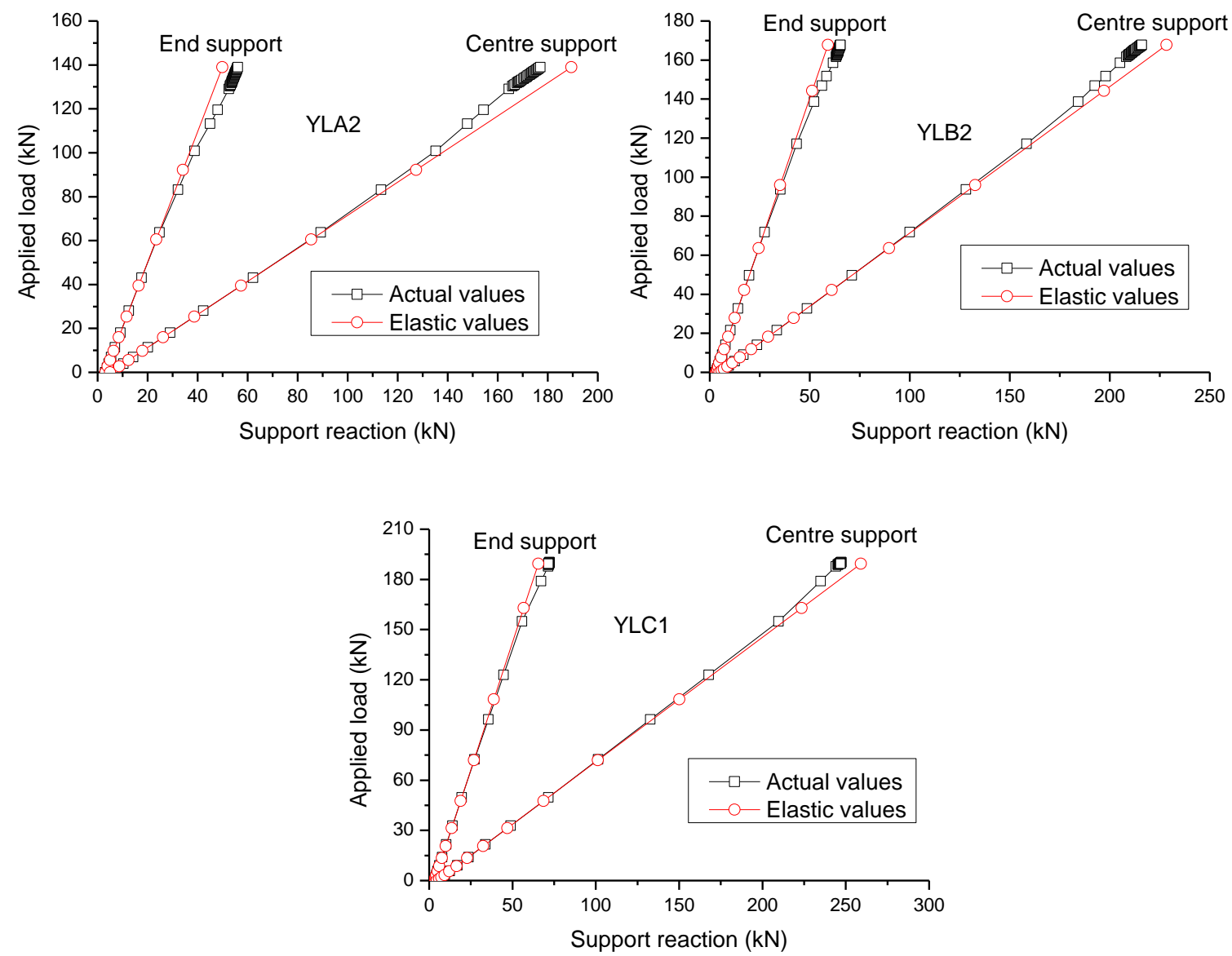

Fig. 11 Support reaction evolution for the continuous beams 

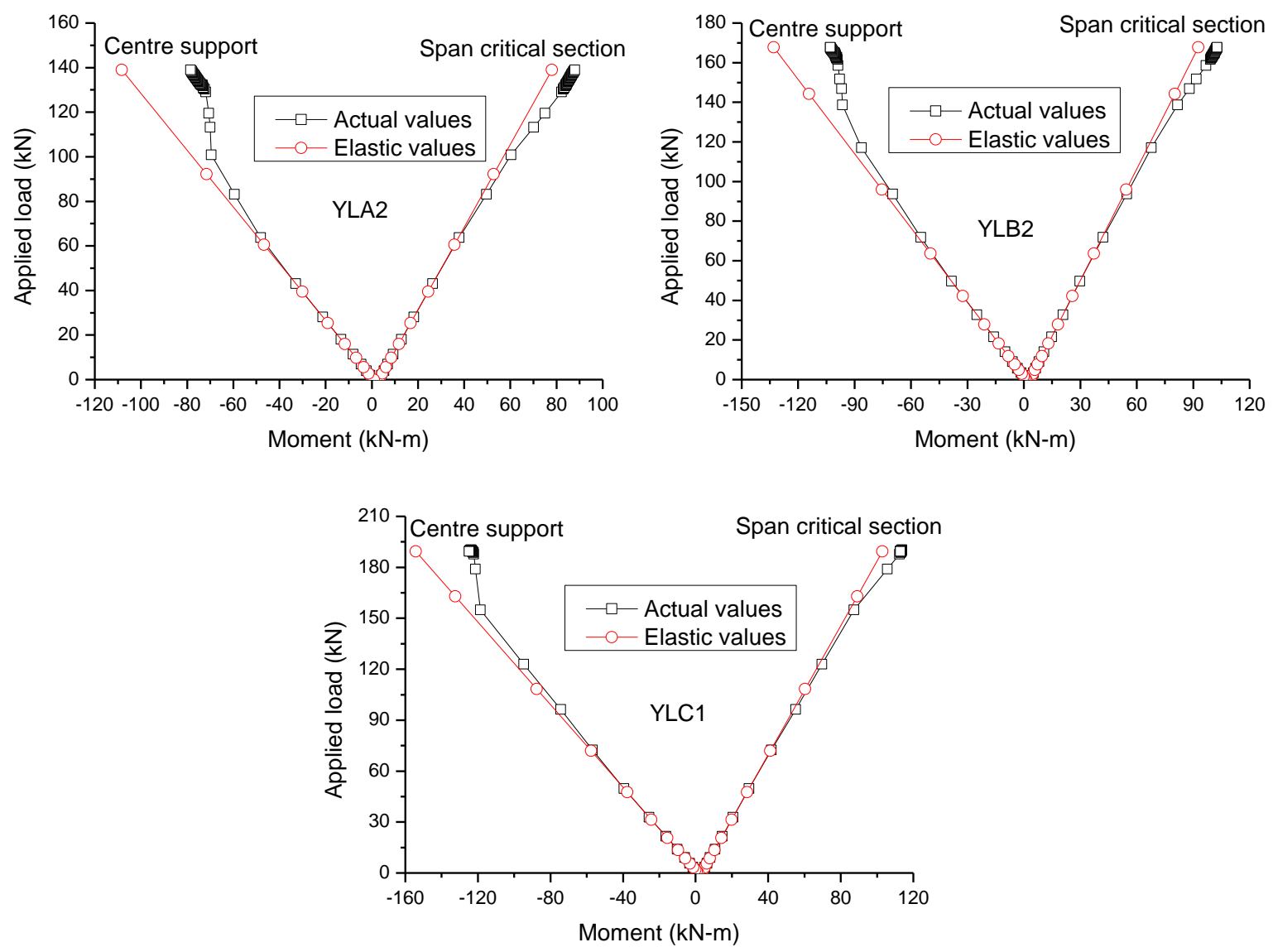

Fig. 12 Moment evolution for the continuous beams 

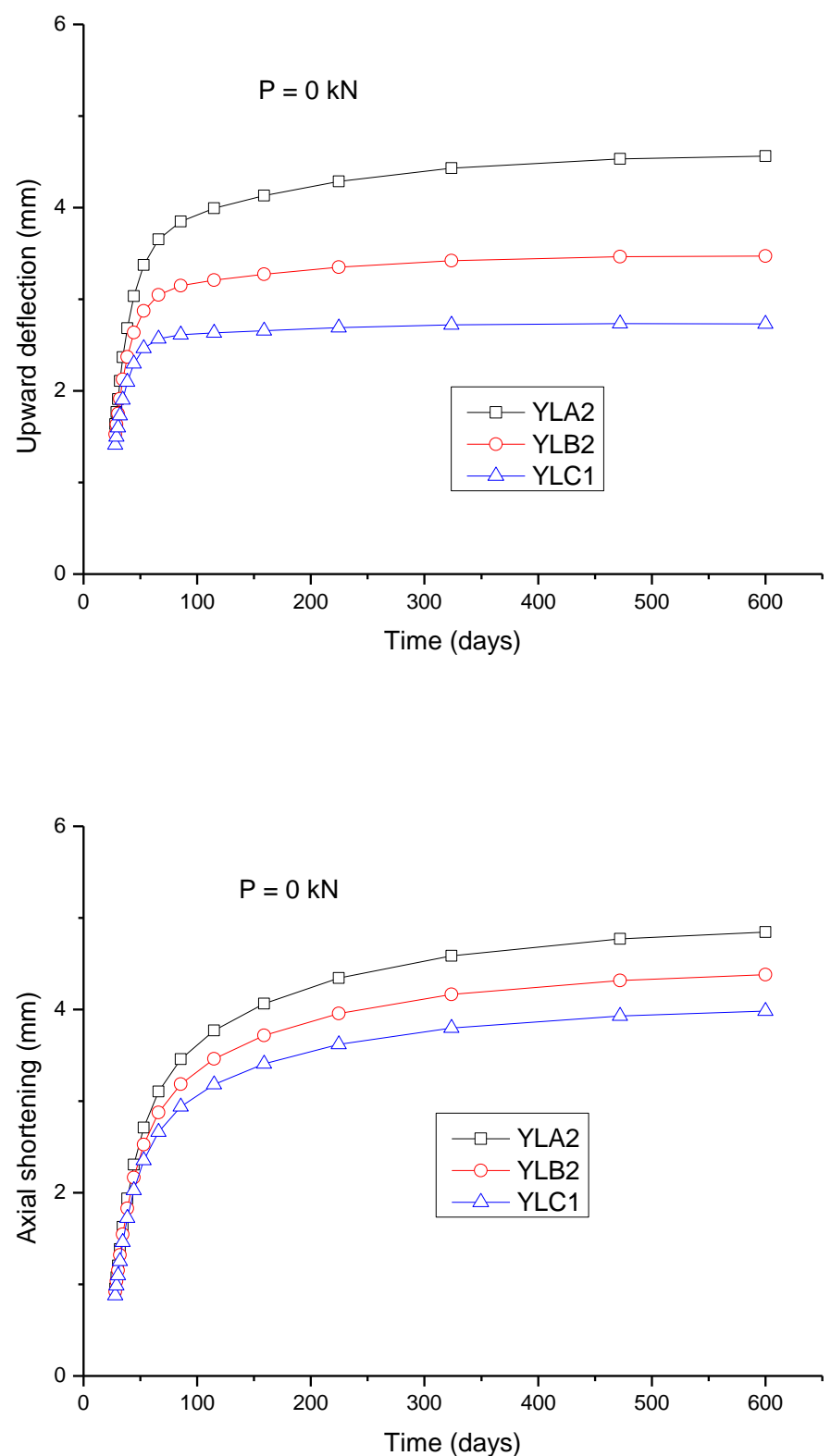

Fig. 13 Time-dependent deformations for the continuous beams under zero live load 

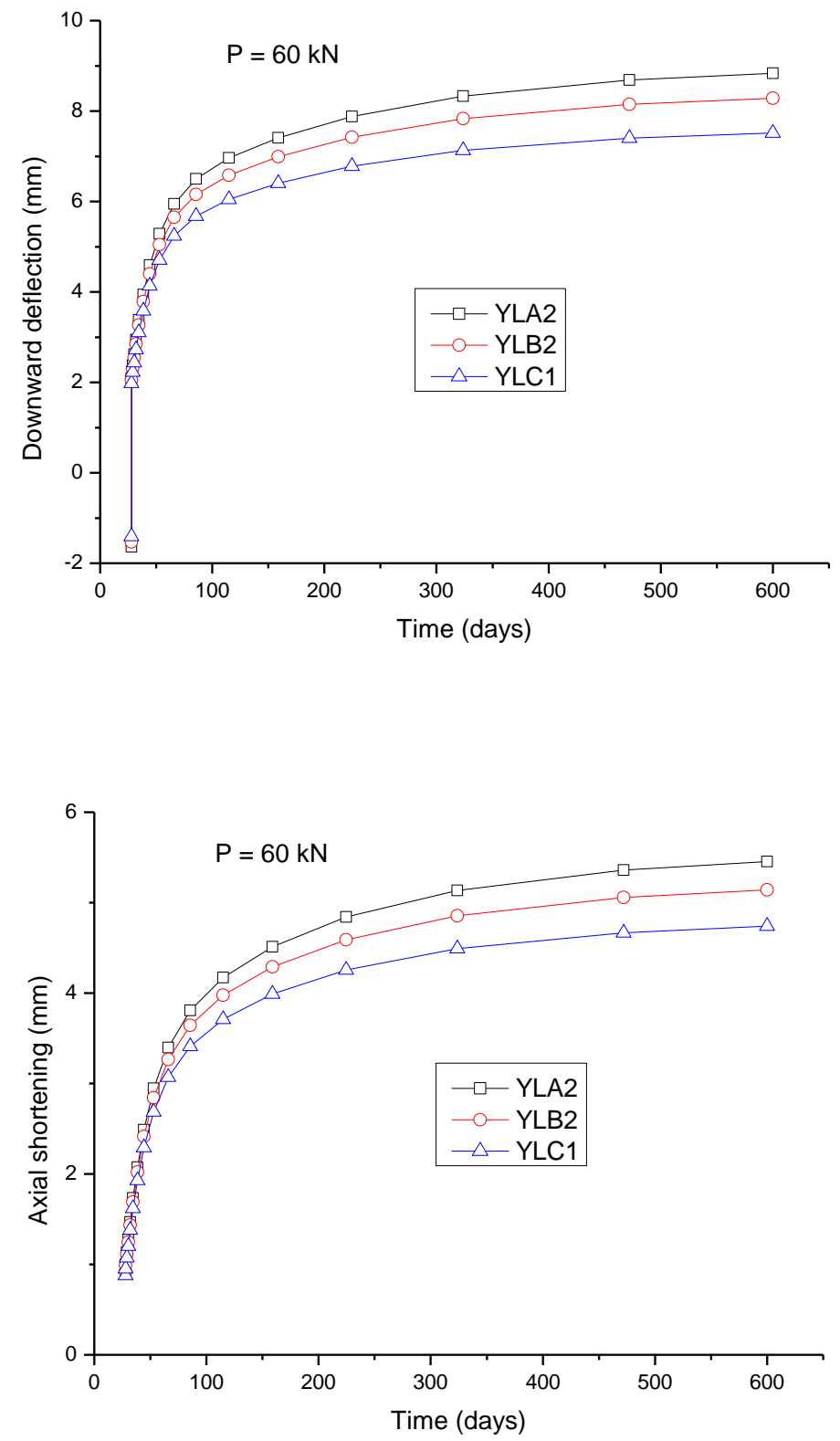

Fig. 14 Time-dependent deformations for the continuous beams under live loading 